\title{
Advances on Manufacturing of POSS Reinforced Resin Matrix Composites
}

\author{
Dan-Yong WANG ${ }^{1, a}$, Jun-Cong LIU ${ }^{1, b}$, Yi-Wei CHEN ${ }^{1}$, Shu-Hu LI ${ }^{1}$, \\ Hua-Zhen $\mathrm{WEI}^{1}$, Min $\mathrm{XIA}^{1}$ \\ ${ }^{1}$ NORINCO GROUP Institute 53, Jinan 250031, China \\ a13356656553@189.cn, b123liujuncong@163.com
}

Keywords: POSS, Manufacturing, Blending, Grafting, Cross-linking, Copolymerization.

\begin{abstract}
POSS with cage-like hybrid molecules composed of silicon and oxygen has raised great interests of materials researchers owing to its excellent mechanical and thermal properties, such as tensile strength, tensile modulus and glass transition temperature $\mathrm{Tg}$. And it has been widely used in electronics, automotives and multiple applications. The four basic manufacturing advances on POSS reinforced resin matrix composites were revealed in this paper and the corresponding conclusions were given. The advances concluded that POSS as reinforced phase could to some extent improve the mechanical and thermal properties of nanocomposites.
\end{abstract}

\section{Introduction}

Polyhedral oligomeric silsesquioxanes (POSS) is cage-like hybrid molecules composed of silicon and oxygen, ranging in size from approximately 1 to $3 \mathrm{~nm}$ with some organic $\mathrm{R}$ groups and once it was discovered, it has quickly raised the increasing interests of scientists in air force and other laboratories science 1990s[1]. Numerous studies proved that the incorporation of POSS into polymers could to some extent enhance mechanical properties. At the same time, POSS reinforced polymers could to some extend modify or improve glass-transition temperature of the base resin enhanced upon copolymerization with associated POSS monomer. The modified materials could be used for advanced electronics, aerospace, automotive and other multiple applications[2,3].

POSS as a nanosized, cage-shaped, three-dimensional structure could be incorporated into almost all kinds of thermoplastic or thermosetting polymers by blending, grafting, cross-linking or copolymerization, in order to modify their mechanical and thermal properties and oxidation resistance, and to reduce their flammability $[4,5]$.

\section{Manufacturing Methods}

\section{Blending}

Zhang[6] prepared organic-inorganic hybrid nanocomposites from a resole phenolic resin and a POSS mixture containing $0.95 \mathrm{wt} \%$ trisilanolphenyl POSS was investigated. Composites with 1.0-10.4 wt $\%$ of POSS were also prepared for comparison. TGA measurements in air confirmed that the temperature for $5 \%$ mass loss increases with increasing of POSS loading.

The chemical incorporation of POSS silanols into methylsilicone networks was investigated by Liu et al[7]. Four kinds of POSS molecules were blended into silanol-terminated methylsilicone resin before end-linking. TGA showed that the thermal stability was greatly improved by POSS.

A study of PEO with melt blended POSS in the whole composition range was conducted by Huang[8]. The POSS moieties included OS-POSS, OA-POSS, OP-POSS. The composites showed two distinct glass transition steps in DSC, neither significantly altered as a function of relative composition. The other two species had Tg significantly higher than that of the polymer.

Shibata[9] synthesized a POSS moiety with average three pyridyl and five phenyl vertex groups and then solution-blended it with pristine and sulfonated polystyrene. Addition of POSS significantly suppressed the glass transition step, especially for high sulfondtion levels.

$\mathrm{Xu}[10]$ incorporated POSS into epoxy system either pre-reacted or non-reacted using 
hexahydrophthalic anhydride (HHPA) as curing agent. And then Non-reacted POSS/epoxy hybrid materials were prepared by directly mixing AH-POSS, HHPA and DGEBA together and cured afterwards. The results showed that $\mathrm{Tg}$ and modulus irregularly decreased.

Teo[11] analyzed novel epoxy-based hybrids prepared via incorporating POSS-IMC and POSS-MMT into the resin based on ECHM and HHPA. The result showed that both POSS-IMC and POSS-MMT could reduce the cure temperature of the epoxy/anhydride system.

The pyrolysis and fire behavior of EP composites based on a novel POSS containing DOPO-POSS and DGEBA were investigated by Zhang[12]. Analysis indicated that the DOPO-POSS changed decomposition pathways of the epoxy resin and increased its residue at high temperature.

Zhang[13] prepared epoxy resins by thermal curing and dispersed DOPO-POSS into DGEBA by mechanical stirring. And the curing agent ( $\mathrm{m}$-phenylenediamine) were then added and mixture were cured and post-cured. Nanocomposites with $2.5 \mathrm{wt} \%$ DOPO-POSS obtained best properties.

Flame retarded polylactide with pentaerythritol phosphate (PEPA), melamine phosphate (MP) and POSS were prepared by Song et al[14]. The results revealed that the UL-94 V-0 materials were obtained with a formulation of $20 \mathrm{wt} \%$ IFR and $5 \mathrm{wt} \%$ TPOSS.

Wang[15] modified UV-curable epoxy acrylate (EA) with OMP-POSS was prepared via thiol-ene photopolymerization. The result of thermal degradation of the cured films showed that OMP-POSS had a double effect on the thermal stability of EA.

Novel PSMA-POSS nanocomposites were prepared by Chen[16], in which N-phenylaminomethyl POSS was conducted as cross-linking agent to incorporate to PSMA. POSS was introduced by one-step reactive solution blend in amounts of $30 \mathrm{wt} \%$. DSC results showed that $\mathrm{Tg}$ increased to $168^{\circ} \mathrm{C}$ with the increase of POSS loading.

EA coating modified with MAP-POSS was prepared by Gao[17]. These results showed Tg increased with increasing MAP-POSS content. When MAP-POSS content is $12 \mathrm{wt} \%$, the Tg reached the maximum $54.3^{\circ} \mathrm{C}$ which was $9.5^{\circ} \mathrm{C}$ higher than that of pure epoxy acrylate.

Montero[18] prepared organic-inorganic by incorporation of POSS(TSP), POMS(AlP) and an organoclay (OC), in an epoxy-amine system. Results showed that POSS addition improved the flame retardancy. The result showed that hybrid with $3 \mathrm{wt} \%$ AlP showed good thermal stability.

Zhang[19] prepared PH/POSS/PMHS/ASR composites. The results revealed that the thermal stabilities and mechanical properties of ASR were significantly enhanced by the addition of PH.

Tucker[20] modified a room-temperature cure epoxy consisting of DGEBF and DETA with $26.5 \mathrm{wt} \%$ and $63.5 \mathrm{wt} \%$ POSS by solvent-blending. The results showed that an increase of more than $300 \%$ in $150{ }^{\circ} \mathrm{C}$ storage modulus and more than $50 \%$ in tensile modulus were observed.

The synthesis and thermal characterization of several POSS modified polyurethane elastomers by Lewicki[21]. The results demonstrated that low levels of POSS substitution (lower than 10wt\%) led to a significant increase in both the onset temperature of thermal de-polymerization.

Durmus[22] Introduced methyl-POSS particles in polyoxymethylene (POM) phase via melt-blending of i-PP nanocomposite as POSS carrier material with POM in a twin screw co-rotating extruder.

Martins[23] prepared nanocomposites of poly(vinylidene fluoride) containing POSS through melt blending. The result showed that nanocomposite with $5 \mathrm{wt}$ \% content had the highest values for degree of cristallinity.

Chen[24] prepared POSS (DVPS) as an octavinyl-POSS derivative first and then series of novel polydimethylsiloxane (PDMS)/DVPS hybrid materials as room temperature vulcanized silicone rubber were prepared. Results significantly enhanced effects on thermal and mechanical properties.

Florea et al[25] synthesized two types of POSS based DGEBA/DG-PDMS nanocomposites were synthesized. The results showed that POSS based nanocomposites enhanced thermo-mechanical properties were obtained for those reinforced with OEP-POSS.

Andrade[26] blends nylon 6 and APOSS which contained 0-5\% POSS by weight. APOSS was previously demonstrated to be an ineffectual additive at concentrations of more than approximately $3 \%$ by weight, lacking solubility and favorable interactions with the polymer matrix. 
The morphology and thermo-mechanical behaviour of nanocomposites formed by PC matrix and POSS with phenethyl substituents (Ph-POSS) were studied by Soto[27]. The Ph-POSS nanocages were added to the PC by melt-blending at loadings between $0 \%$ and $15 \mathrm{wt} \%$. DSC and DMA analyses showed that increasing amount of nanocages caused continuous decrease on Tg.

Sanchez-Soto[28] studied melt blends of different POSS species in POM. Moieties with eight PEO substituents and epoxidized cages with seven ethyl substituents did not disperse well.

Zhou[29] added dicumyl peroxide to facilitate reactive blending with the matrix. The dispersion of POSS dramatically improved with aggregates shrinking from $200-1000 \mathrm{~nm}$ to $50-200 \mathrm{~nm}$. The hybrids also improved modulus due to cross-linking. But Tg decreased by a few degrees.

Most of references proved that POSS reinforced resin composites could improve Tg with POSS content of $0-10 \mathrm{wt} \%$ or $5 \mathrm{wt} \%$ POSS reinforced composites could be up to the top point, while mechanical property with $0-30 \mathrm{wt} \%$.

\section{Co-polymerization}

Such POSS moieties with seven cyclopentyl or i-butyl vertex groups were copolymerized with propylene by Zhang[30]. TEM showed that i-butyl-POSS disperse better in the matrix. Both species had significantly increased calorimetric Tg, but i-butyl POSS had a more prominent effect.

Seurer and Coughlin[31] prepared such moieties with seven phenyl, isobutyl, isooctyl or ethyl inert vertex groups and incorporated them in a copolymer of ethylene and propylene with POSS as high as 30wt $\%$. Olefine-POSS act as plasticizers reduced Tg but phenyl-POSS slightly increased .

Similar results were reported in the case of PET polymerized in the presence of disilanol and trisilanol i-butyl-POSS[32]. The results showed that up to $3 \mathrm{wt} \%$ dispersed well to $30-40 \mathrm{~nm}$ structures at higher loadings tended to aggregate. Both viscosity and complex modulus increased.

Blanco[33,34] followed the in-situ polymerization approach, with up to $10 \mathrm{wt} \%$ of phenyl heptacyclopentyl. Tg for various polystyrene-POSS systems prepared with particles present during polymerization $^{[33]}$ or by melt blending[35]. In this case, no effect of POSS on Tg was observed.

Florea studied IPN based on dimethacrylic/epoxy resins via in-situ polymerization reinforced with octafunctional POSS (with methacrylate or epoxy groups)[36]. The results proved that the incorporation of OEP-POSS within epoxy resin led to a decrease of thermo-mechanical properties.

Above were researches of POSS with content from 0-30wt\% reinforced nanocomposite and the results proved that it could to some extent improve its mechanical and thermal properties, but POSS reinforced epoxy resin composites on the contrary.

\section{Grafting}

Bianchi[37] grafted POSS on PS chains by reactive blending, using DCP as a free radical initiator. And the result revealed that a degree of hybridization up to $40 \%$ was achieved. A moderate increase in Tg was observed, that at the highest gross amount of $5 \mathrm{wt} \%$ POSS, was $\Delta \mathrm{Tg}=+4.5 \mathrm{~K}$.

PBO fibers was treated through surface grafting of POSS by Song[38]. The effect of POSS grafting on bulk mechanic property and interfacial property of PBO fiber were studied. The results showed that interfacial shear strength between treated PBO fibers and epoxy resin increased to 54.9MPa comparative with untreated one. Tensile strength of treated PBO fiber little decreased.

Jiao[39] synthesized POSS-MPS by the reaction of $\mathrm{NH}_{2}$-MPS with G-POSS which was employed as reinforcing agents to prepare POSS-MPS/EP nanocomposites. Its thermal properties and mechanical properties of the POSS-MPS/EP improved.

The grafting of amino-POSS on CFs surface was achieved through the reaction of the SPDPC grafted on the CFs surface by Jiang[40]. Grated SPDPC and grafted with a combined SPDPC and amino-POSS improved the interfacial performance of the CFs/UPR composites respectively.

\section{Cross-linking}

Chen[41] firstly introduced both DVPS and PDMS as the cross-linker and the reinforcing filler respectively. The results exhibited that the thermal stabilities and mechanical properties of the novel RTV silicone rubbers were far better than those of the reference materials (DVPR and MTFR).

In the past five years, Chen et al were undergoing researches of cross-linking POSS to matrix 
materials and experiment results exhibited that by making full use of this method, thermal and mechanical properties of POSS reinforced nanocomposites could be reinforced.

\section{Conclusion}

The fabrication methods of POSS reinforced nanocomposites were introduced in this paper. The basic four methods including blending, co-polymerization, grafting and cross-linking and their corresponding researches advances were reviewed too. The relative conclusions are as follows.

(1) POSS reinforced thermoplastics resin matrix composites could to some extend improve the corresponding mechanical and thermal properties.

(2) Most of references proved that POSS reinforced resin composites could improve Tg with POSS content of $0-10 \mathrm{wt} \%$ or $5 \mathrm{wt} \%$ POSS reinforced composites could make it reach up to the top point, while mechanical property with $0-30 \mathrm{wt} \%$.

(3) The reinforcement effects of POSS nanocomposites to some extend depended on the interfacial surface bonding. In the basic four methods, blending and are convenient to operate but the reinforcement effects are not as well as the other three methods.

\section{References}

[1] A. Romo-Uribe, P. T. Mather, T. S. Haddad, J. D. J. Lichtenhan, Polym. Sci. Part B: Polym. Phys. Vol.36 (1998), p.1857.

[2] T.L. Lu, G.Z. Liang, Y.L. Peng, T. Chen, Journal of Applied Polymer Science. Vol.106(2007), p.4117.

[3] H.Z. Liu, S.X. Zheng, K.M. Nie. Macromolecules. Vol.38(2005), p.5088.

[4] R.H.Baney, M. Itoh, A.Sakakibara, et al. Chem. Rev. Vol.95(1995), p.1409-1430.

[5] H.Z. Liu, W.A. Zhang, S.X. Zheng. Montmorillonite intercalated by Ammonium of Octa-aminopropyl Polyhedral Oligomeric Silsesquioxane and its Nanocomposites with Epoxy Resin. Polymer. Vol.46(2005), p.157-165.

[6] Y.D. Zhang, S.H. Lee, M. Yoonessi, et al. Phenolic Resin-trisilanolphenyl Polyhedral Oligomeric Silsesquioxane (POSS) Hybrid Nanocomposites: Structure and Properties. Vol.47(2006), p.2984-2996.

[7] Y.R. Liu, Y.D. Huang, L. Liu. Thermal Stability of POSS/Methylsilicone Nanocomposites. Composites Science and Technology. Vol.67(2007), p.2864-2876.

[8] K.W. Huang, L.W.Tsai, S.W. Kuo. Influence of Octakis-functionalized Polyhedral Oligomeric Silsesquioxanes on the Physical Properties of Their Polymer Nanocomposites. Polymer. Vol.50(2009), p.4876-87.

[9] M. Shibata, R. Horie, W. Yoneta. Intermolecular Interaction of Supramolecular Organicinorganic Hybrid Composites of Sulfonated Polystyrene and Oligomeric Silsesquioxane Possessing Pyridyl Groups. Polymer. Vol.51(2010), p.5764-5770.

[10] Y.T. Xu, Y.Y. Ma, Y.M. Deng, et al. Morphology and Thermal Propertis of Organic-inorganic Hybrid Material Involving Monofunctional-anhydride POSS and Epoxy Resin. Materials Chemistry and Physics. Vol.125(2011):174-183.

[11] J.K. Herman Teo, C.L. Toh, X.H. Lu. Catalytic and Reinforcing Effects of Polyhedral Oligomeric Silsesquioxane(POSS)-imidazolium Modified Clay in an Anhydride-cured Epoxy. Vol.52(2011), p.1975-1982.

[12] W.C. Zhang, X.M. Li, R.J. Yang. Pyrolysis and Fire Behaviour of Epoxy Resin Composites Based on a Phosphorus-containing Polyhedral Oligomeric Silsesquioxane (DOPO-POSS). Polymer 
Degradation and Stability. Vol.96(2011), p.1821-1832.

[13] W.C. Zhang, X.M. Li, R.J. Yang. Novel Flame Retardancy Effects of DOPO-POSS on Epoxy Resins. Polymer Degradation and Stability, Vol.96(2011), p.2167-2173.

[14] L. Song, S.Y.Xuan, X. Wang, et al. Flame Retardancy and Thermal Degradation Behaviors of Phosphate in Combination with POSS in Polylactide Composites. Thermochimica Acta. Vol.527(2012), p.1-7.

[15] X.F. Wang, X. Wang, L. Song, et al. Preparation and Thermal Stability of UV-cured Epoxy-based Coatings Modified with Octamercaptopropyl POSS. Thermochimica Acta. Vol.568(2013), p.130-139.

[16] P.P. Chen, X. Huang, Q.H. Zhang, et al. Hybrid Networks Based on Poly(styrene-co-maleic anhydride) and N-phenylaminomethyl POSS. Polymer. Vol.54(2013), p.1091-1097.

[17] J.G.g Gao, H.Q. Lv, X.F. Zhang, et al. Synthesis and Properties of Waterborne Epoxy Acrylate Nanocomposite Coating Modified by MAP-POSS. Progress in Organic Coatings. Vol.76(2013), p. 1477-1483.

[18] B. Montero, R. Bellas, C. Ramirez, et al. Flame Retardancy and Thermal Stability of Organic-inorganic Hybrid Resins Based on Polyhedral Oligomeric Silsequioxanes and Montmorillonite Clay. Composites: Part B. Vol.63(2014), p.67-76.

[19] Y.F. Zhang, Y.Y. Mao, D.Z. Chen, et al. Synthesis and Characterization of Addition-type Silicone Rubbers (ASR) Using a Novel Cross Linking Agent PH Prepared by Vinyl-POSS and PMHS. Polymer Degradation and Stability. Vol.98(2013), p.916-925.

[20] S.J. Tucker, B. Fu, S. Kar, et al. Ambient Cure POSS-epoxy Matrices for Marine Composites. Composites: Part A. Vol.41(2010), p.1441-1446.

[21] J.P. Lewicki, K. Pielichowski, M. Jancia, et al. Degradative and Morphological Characterization of POSS Modified Nanohybrid Polyurethane Elastomers. Polymer Degradation and Stability, Vol.104(2014), p.50-56.

[22] A. Durmus, A. Kasgoz, N. Ercan, et al. Effect of Polyhedral Oligomeric Silsesquioxane (POSS) Reinforced Polypropylene (PP) Nanocomposite on the Microstructure and Isothermal Crystallization Kinetics of Polyoxymethylene (POM). Polymer, Vol.53(2012), p.5347-5357.

[23] J.N. Martins, T.S.Bassani, V.B.Oliveira. Morphological, Viscoelastic and Thermal Properties of Poly(vinylidene Fluoride)/POSS Nanocomposites. Materials Science and Engineering C. Vol.32(2012), p:146-151.

[24] D.Z. Chen, J.R. Nie, S.P. Yi, et al. Thermal Behavior and Mechanical Properties of Novel RTV Silicone Rubber Using Divinyl-hexa[(trimethoxysilyl)ethyl]-POSS as Cross-linker. Polymer Degradation and Stability, Vol.95(2010), p.618-626.

[25] N.M.Florea, A.Lungu, P.Badica, et al. Novel Nanocomposites Based on Epoxy Resin/Epoxy-functionalized Polydimethysiloxane Reinforced with POSS. Composites Part B. Vol.75(2015), p.226-234.

[26] R.J. Andrade, R.Z. Huang, M.M. Herbert, et al. A Thermo-rheological Study on the Structure Property Relationships in the Reinforcement of Nylon 6-POSS Blends. Polymer. Vol.55(2014),p.860-870.

[27] M. Sánchez-Soto, D.A. Schiraldi, S. Illescas. Study of the Morphology and Properties of Melt-mixed Polycarbonate-POSS Nanocomposites. European Polymer Journal, Vol. 45(2009), p.341-352. 
[28] S.M. Soto, S. Illescas,, H. Milliman,, D.A. Schiraldi,, A.Arostegui. Morphology and Thermomechanical Properties of Melt-mixed Polyoxymethylene/polyhedral Oligomeric Silsesquioxane Nocomposites. Macromol Mater Eng. Vol.295(2010), p.846-58.

[29] Z. Zhou, L. Cui, Y. Zhang, N. Yin. Preparation and Properties of POSS Grafted Polypropylene by Reactive Blending. Eur Polym J. Vol.44(2008), p.3057-66.

[30] H.X.Zhang, Y.J. Shin, K.B.Yoon., D.H. Lee.. Preparation and Properties of Propylene/POSS Copolymer with Rac-Et(Ind) ${ }_{2} \mathrm{ZrCl}_{2}$ Catalyst. EurPolym J. Vol.45(2009), p.40-46.

[31] B. Seurer, E.B. Coughlin. Ethylene-propylene-silsesquioxane Thermoplastic Elastomers. Macromol Chem Phys. Vol.209(2008), p.1198-209.

[32] J.K. Kim, K.H. Yoon, D.S. Bang, Y.B. Park, H.U. Kim, et al. Morphology and Rheological Behaviors of Poly(ethylene terephthalate) Nanocomposites Containing Polyhedral Oligomeric Silsesquioxanes. J Appl Polym Sci. Vol.107(2008), p.272-279.

[33] I. Blanco, L. Abate, F.A. Bottino, P. Bottino. Thermal Degradation of Hepta Cyclopentyl, Mono Phenyl-polyhedral Oligomeric Silsesquioxane (hcp-POSS)/Polystyrene (PS) Nanocomposites. Polym Degrad Stab. Vol.97(2012), p.849-55.

[34] I. Blanco, F.A. Bottino. Thermal Study on Phenyl, Hepta Isobutylpolyhedral Oligomeric Silsesquioxane/Polystyrene Nanocomposites. Polym Compos. Vol.34(2013), p.225-232.

[35] N.T. Dintcheva,, E. Morici,, R. Arrigo., La Mantia FP, Malatesta V, Schwab JJ. Structure-properties Relationships of Polyhedral Oligomeric Silsesquioxane (POSS) Filled PS -nanocomposites. Express Polym Lett. Vol.6(2012), p.561-571.

[36] Lungu A., Florea NM, Lovu H. Dimethacrylic/Epoxy Interpenetrating Polymer Networks Including Octafunctional POSS. Polymer. Vol. 53(2), 2012, p.300-307.

[37] O. Bianchi, L.L. Barbosa, G. Machado, L.B. Canto, R.S. Mauler, R.V.B. Oliveira. Reactive Melt Blending of PS-POSS Hybrid Nanocomposites. J. Appl Polym Sci. Vol. 128(2013), p.811-827.

[38] B. Song, L.H. Meng, Y.D. Huang. Improvement of Interfacial Property Between PBO Fibers and Epoxy Resin by Surface Grafting of Polyhedral Oligomeric Sillsesquioxanes (POSS). Applied Surface Science. Vol. 258(2012), p.10154-10159.

[39] J. Jiao, L. Wang, P.P. Lv, et al. Improved Dielectric and Mechanical Properties of Silica/epoxy Resin Nanocomposites Prepared with a Novel Organic-inorganic Hybrid Mesporous Silica: POSS-MPS. Materials Letters, Vol. 129(2014), p.16-19.

[40] D.W. Jiang, L. Liu, J. Long, et al. Reinforced unsaturated polyester composites by chemically grafting amino-POSS onto carbon fibers with active double spiral structural spiral phosphodicholor. Composites Science and Technology, Vol. 100(2014), p.158-165.

[41] D.Z. Chen, Y. Liu, C. Huang. Synergistic Effect Between POSS and Fumed Silica on Thermal Stabilities and Mechanical Properties of Room Temperature Vulcanized (RTV) Silicone Rubbers. Polymer Degradation and Stability, Vol.97(2012), p.308-315. 